\title{
Diabetes: stem cells offering healthy promises
}

\section{Editorial}

Diabetes is a chronic lifelong disease and according to Diabetes Association of America, in 2015 itself approximately 30.3million Americans (9.4\% of the population) have the disease. Unfortunately, almost one fourth (or approximately 7.2million) are unaware that they have it. An additional 84.1 million people have pre-diabetes. With increasing prosperity, its prevalence has increased in almost all populations of the world and ranges from 5-15\%. As it affects so big portion of the world population a long-lasting cure is urgently warranted. People with diabetes need to manage their disease in order to avoid related complications and maintain healthy social and economic interactions.

Diabetes affects individuals of all age groups and has been classified in two types. Type 1 diabetes (T1D) is an autoimmune disease that occurs when a person's pancreas stops producing insulin. It is usually diagnosed in children and young adults, previously known as juvenile diabetes. Only 5\% of people with diabetes have this form of the disease. On the other hand, type 2 diabetes (T2D) is the most common form of diabetes. In patients of T2D, the body does not use insulin properly mostly because of insulin resistance. Because of that, at first, pancreas compensates by making extra insulin. However, over time it isn't able to keep up and can't make enough insulin to keep your blood glucose at normal levels.

Diabetes affects every part of the body and causes complications related to heart, brain, kidney, circulatory system etc. Managing diabetes exerts a significant burden on the economy in general. During 2017, according to an estimate, diabetes-related care of people directly or indirectly could have costed around \$327 billion. ${ }^{1}$ Though a number of medications are already in clinical use but none of them grant a long-term cure and all of them have some or other undesired side-effects.

Since almost all pharmacological drugs, irrespective of the target molecule in the pathway involved in the manifestation of diabetesrelated complications, have some side effects a safer and comparatively long last therapeutic alternative is desperately needed. To meet the continuous need for insulin, pancreatic transplants have been tried which turned out to be very cost-intensive and impractical because the donor pancreases have to be recovered from suitable cadavers and then transplanted. ${ }^{2}$ Logically, transplantation of a tissue from other individual comes up with so many concerns like compatibility of a graft in the new host and its survival as immune rejection is usually a valid concern most of the time. To circumvent all these concerns another alternative way of handling the problem was needed for a long time. Discovery of stem cells and related extensive research has offered a ray of hope to manage the problem efficiently with a sound possibility of a permanent cure.

Stem cells, depending on the source of their origin, are classified as embryonic or adult or induced. Embryonic stem cells are capable of differentiating in all cell types for a body while adult cells which have
Volume 4 Issue 2 - 2018

\author{
Aditi Saraswat,' Anand Srivastava ${ }^{2}$ \\ 'Henry Ford Medical Center, USA \\ ${ }^{2}$ Global Institute of Stem Cell Therapy and Research, USA
}

Correspondence: Anand Srivastava, Global Institute of Stem Cell Therapy and Research, 4660 La Jolla Village Drive, San Diego, CA, 92122, USA, Tel 85834424 92, Email anand@giostar.com

Received: April 30, 2018| Published: May 09, 2018

attained some tissue-specific differentiation, lose that ability slightly. Since a number of ethical issues crop up with use of embryonic cells, adult stem cells are next best type of cells to lean back on. Another favorable factor for using adult stem cells is that these can be isolated from tissues which are easy to extract from an individual like belly fat or bone marrow. Cells of these origins are classified as mesenchymal stem cells (MSCs). MSCs are known to promote the regeneration of pancreatic islet beta cells, protect endogenous pancreatic islet beta cells from apoptosis, and ameliorate insulin resistance of peripheral tissues by providing a supportive niche microenvironment driven by the secretion of paracrine factors or the deposition of extracellular matrix. ${ }^{3,4}$

In general, implantation of MSCs can alleviate T2D by a number of mechanisms. These cells, if implanted directly in the pancreas, thanks to their multipotential ability to differentiate in diverse types of cells of their immediate vicinity, can produce new insulin-producing cells. Investigators, in order to promote the chance of differentiation of cells in insulin-producing cells, have preprogrammed MSCs by culturing in serum-free high glucose media or neuron conditioned media before transplantation. Intravenous infusion of stem cell has been shown to regenerate beta cells of islets in rats ${ }^{5}$ also promote the survival during hypoxia and oxidant stress. ${ }^{6}$ In addition to these effects, infusion of stem cells has been shown to promote insulin sensitivity. ${ }^{7}$ Though the exact mechanism by which stem cell bring about increased insulin sensitivity is not deciphered, it could be because of stem-cell-mediated decrease in systemic inflammation as it is well established that insulin resistance is strongly correlated with chronic low-grade inflammation.

Encouraging findings in cases of diabetes treatments with stem cell therapies have led the clinicians to try implantation or infusion or both in the clinical set up also. On the clinical trial site of NIH, more than 150 trials at different stage have been listed. MSCs of diverse origins either were implanted directly in the pancreas ${ }^{8}$ or were infused in blood stream ${ }^{9}$ or both ${ }^{10}$ showed promising results up to 12 months of follow up. A couple of clinical parameters are often used to ascertain the effectiveness of a therapy in cases of diabetes. A decrease in $\mathrm{Hb}$ $\mathrm{A} 1 \mathrm{C}$ is one of those parameters which were used by Estrada et al. ${ }^{11}$ and they reported a significant decrease..$^{11}$ In another study, insulin need decreased or was abolished altogether. ${ }^{8}$ Similarly, implantation or infusion of MSCs has been shown to improve pancreatic function i.e. 
increased insulin production. Same time, increased insulin sensitivity is also attained by MSCs.

Just like other medical helps, stem cell therapy can have some undesirable effects, though the incidences are few and far between. Even those undesired effects, which happen after stem cell transplantation are very mild and easily manageable like mild to moderate fever or nausea or headache.

In conclusion, stem cell therapy does offer a long lasting therapeutic alternative for treating T2D. Same time it has to be kept in minds of both clinicians and patients that it is not a permanent cure. T2D is a metabolic syndrome which manifests after a long duration of unhealthy life style which needs to be addressed in order to lead a healthy life. Compared to all other available therapies, stem cell therapy can offer a lot longer period for individuals to develop a healthy life style which would help fend off reoccurrence of the disease.

\section{Acknowledgements}

None.

\section{Conflict of interest}

The authors declare that there is no conflict of interest.

\section{References}

1. Enocrinology Advisor. Total Estimated Cost of Diagnosed Diabetes $\$ 327$ Billion in 2017. Endocrinology Advisor. 2017.

2. Shapiro AM, Lakey JR, Ryan EA, et al. Islet transplantation in seven patients with type 1 diabetes mellitus using a glucocorticoid-free immunosuppressive regimen. $N$ Engl J Med. 2000;343(4):230-238.

3. Caplan AI, Dennis JE. Mesenchymal stem cells as trophic mediators. $J$ Cell Biochem. 2006;98(5):1076-1084.
4. Lee RH, Seo MJ, Reger RL, et al. Multipotent stromal cells from human marrow home to and promote repair of pancreatic islets and renal glomeruli in diabetic NOD/scid mice. Proc Natl Acad Sci U S A. 2006;103(46):17438-17443.

5. Hao H, Liu J, Shen J, et al. Multiple intravenous infusions of bone marrow mesenchymal stem cells reverse hyperglycemia in experimental type 2 diabetes rats. Biochem Biophys Res Commun. 2013;436(3):418-423.

6. Chandravanshi B, Bhonde RR. Shielding Engineered Islets With Mesenchymal Stem Cells Enhance Survival Under Hypoxia. J Cell Biochem. 2017;118(9):2672-2683.

7. Hughey CC, Ma L, James FD, et al. Mesenchymal stem cell transplantation for the infarcted heart: therapeutic potential for insulin resistance beyond the heart. Cardiovasc Diabetol. 2013;12:128.

8. Bhansali A, Asokumar P, Walia R, et al. Efficacy and safety of autologous bone marrow-derived stem cell transplantation in patients with type 2 diabetes mellitus: a randomized placebo-controlled study. Cell Transplant. 2014;23(9):1075-1085.

9. Jiang R, Han Z, Zhuo G, et al. Transplantation of placenta-derived mesenchymal stem cells in type 2 diabetes: a pilot study. Front Med. 2011;5(1):94-100.

10. Liu X, Zheng P, Wang X, et al. A preliminary evaluation of efficacy and safety of Wharton's jelly mesenchymal stem cell transplantation in patients with type 2 diabetes mellitus. Stem Cell Res Ther. 2014;5(2):57.

11. Estrada EJ, Valacchi F, Nicora E, et al. Combined treatment of intrapancreatic autologous bone marrow stem cells and hyperbaric oxygen in type 2 diabetes mellitus. Cell Transplant. 2008;17(12):1295-1304. 